\title{
Nursing-Mini-CEX Combined with Online and Offline Hybrid Teaching Model Application Research in Basic Nursing Training Teaching
}

\author{
Hongmin Wang*, Yaru Feng, Huan Li \\ Zhengzhou Sias University, Zhengzhou 451150, Henan, China. \\ E-mail: hongmin_wang699@126.com
}

Fund Project: Zhengzhou Sias College's 2019 Education Reform Fund Project: Nursing-Mini-CEX Combined with Online and Offline Hybrid Teaching Model Application Research in Basic Nursing Training Teaching. Project No. 2019JGYB72

Abstract: Objective: To explore the application effects and countermeasures of Nursing-Mini-CEX combined with the online and offline hybrid teaching model in basic nursing training.

Methods: Taking 150 students from the 2017 grade as an example, the effects of traditional teaching mode and NursingMini-CEX combined with mixed model teaching were compared.

Results: The performance of the intervention group combining Nursing-Mini-CEX evaluation and mixed teaching was significantly higher than that of the control group, and the difference was statistically significant $(\mathrm{P}<0.05)$.

Conclusion: The combination of Nursing-Mini-CEX measurement tools and mixed teaching is feasible and effective in basic nursing training.

Keywords: Nursing-Mini-CEX; Mixed Teaching; Basic Nursing Training

For a long time, the clinical training of many nursing majors has been assessed by theoretical examination combined with skill operation, OSCE, SP and other methods ${ }^{[1]}$ which have gradually been introduced into the evaluation of nursing training, but there are certain limitations, and the evaluation focuses too much on the knowledge system, ignoring knowledge understanding and theoretical application. Therefore, based on the Nursing-Mini-CEX evaluation tool, this article explores how to carry out effective training and evaluation in combination with mixed teaching in basic nursing training.

\section{Research objects and methods}

\subsection{Research objects, time and content}

The time span is May 2019-January 2020, that is, the second semester of the second year and the first year of the third year. Through cluster sampling, 150 nursing students of 2017 grade in a university are selected. The content includes basic nursing training items such as bathing in bed, changing bed sheets of bedridden patients, axis turning method, vital signs measurement, sputum suction, enema, urinary catheterization, intravenous infusion, CPR, etc.

\subsection{Specific methods}

Divide 150 students into 6 groups, led by 3 teachers. The intervention group and the control group each have 3 groups, with 25 people in each group. The intervention group uses a mixed teaching mode to combine teaching methods such as case teaching. the control group uses the traditional teaching method that the teacher will teach through the traditional face-to-face teaching, student independent learning and other decentralized teaching processes, while Copyright (C) 2020 Hongmin Wang et al

doi: 10.18282/le.v9i6.1295

This is an open-access article distributed under the terms of the Creative Commons Attribution Non-Commercial License

(http://creativecommons.org/licenses/by-nc/4.0/), which permits unrestricted non-commercial use, distribution, and reproduction in any medium, provided the original work is properly cited. 
incorporating the case teaching method into the traditional teaching model. The training content of the two groups of subjects is the same.

\subsection{Evaluation method}

Both the control group and the experimental group used Nursing-Mini-CEX to evaluate the same teaching effects. Among them, the clinical communication ability scale ${ }^{[2]}$ and the self-study ability scale ${ }^{[3]}$ mainly evaluate the learning achievements of students, and the final questionnaire survey and interview are used to evaluate the quality of training education.

\subsection{Statistical methods}

The SPSS19.0 software was used to describe the counting data by percentage, and the measurement data was subjected to t-test.

\subsection{Quality control}

Construct a unified online teaching resource library and case library to ensure the consistency and accuracy of teacher evaluation. The School of Nursing established the Nursing-Mini-CEX basic nursing training teaching group Superstar Fanya Learning Group and Dingding Group to regularly communicate and interact with clinical nurses. The supervision, continuous improvement and evaluation of the Nursing-Mini-CEX teaching process will be carried out by the supervision team of the clinical teaching and research section of the college.

\section{Intervention results and discussion}

\subsection{Intervention results}

Through the Nursing-Mini-CEX evaluation and measurement table, analyze the advantages of mixed teaching in the control experiment. The abilities of the two groups of subjects and the average scores of the overall evaluation are shown in Figure 1. It can be seen that the students in the intervention group have higher scores in clinical communication, humanistic care, etc. than the control group, and their overall scores also have obvious advantages.

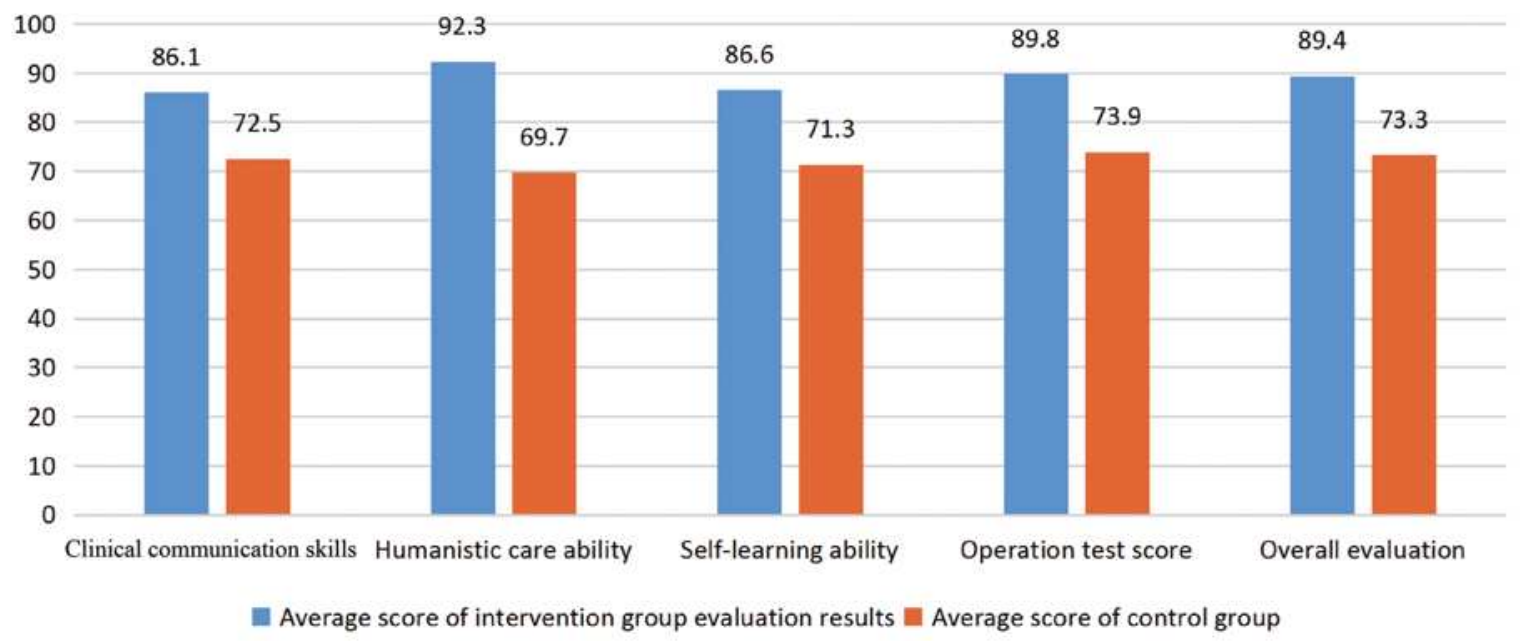

Figure 1. Various abilities and average scores of the overall evaluation.

The samples of the two groups are roughly in line with the normal distribution. The comparison of the data between the two groups is shown in Table 1 . The sub-items of the intervention group and the overall evaluation $\pm \mathrm{S}$ score indicate that the students in the intervention group have smaller and more average differences in performance than the control group. For stability, its mean value is more representative. The overall $\pm \mathrm{S}$ scores of the two groups were $89.4 \pm 8.51$ and $73.3 \pm 6.69$ respectively, which also showed that the distribution of the overall scores of the intervention group students was more precise, and the dispersion of the overall scores of the control group students was more obvious. $\mathrm{P}<0.05$ indicating that there is a significant difference in the basic nursing training situation of the two groups of subjects. Therefore, the learning situation of the intervention group using mixed teaching is significantly better than that of the control group. 
Table 1: Comparison of various abilities and overall evaluation between the two groups $( \pm \mathrm{S})$

\begin{tabular}{|c|c|c|c|c|}
\hline Evaluation item & Intervention group & Control group & $t$ & 3.386 \\
\hline $\begin{array}{c}\text { Clinical communi- } \\
\text { cation }\end{array}$ & $86.1 \pm 7.39$ & $72.5 \pm 6.16$ & 0.001 \\
\hline $\begin{array}{c}\text { Humanistic care } \\
\begin{array}{c}\text { Independent learn- } \\
\text { ing }\end{array}\end{array}$ & $92.3 \pm 8.83$ & $69.7 \pm 5.12$ & 1.982 & 0.052 \\
\hline Operating test & $89.6 \pm 7.42$ & $71.3 \pm 5.25$ & 6.018 & 0.000 \\
\hline Overall score & $89.4 \pm 8.51$ & $73.9 \pm 6.98$ & 6.173 & 0.000 \\
\hline
\end{tabular}

\subsection{Analysis of intervention results and feedback discussion}

After the intervention, students' satisfaction with different teaching methods and assessment methods is shown in Figure 2.

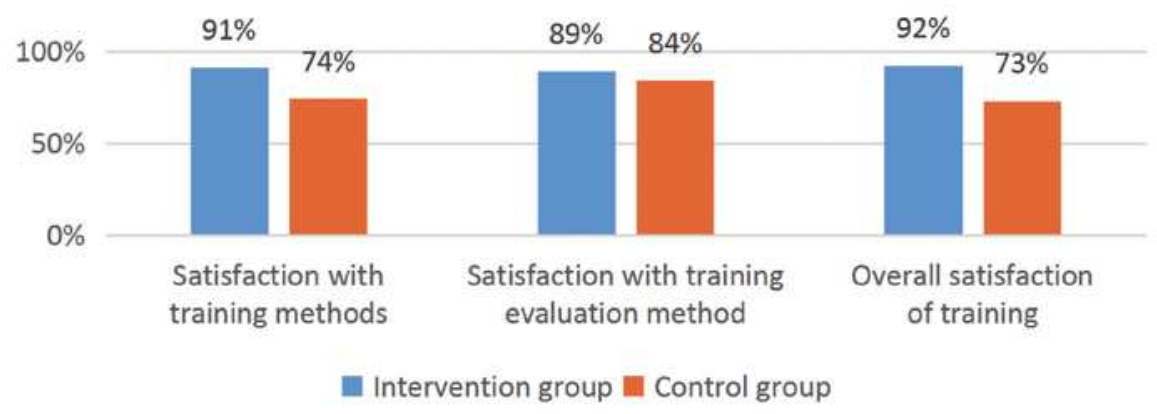

Figure 2. Two groups of training satisfaction surveys

Most of the students in the intervention group believed that blended teaching was better than traditional teaching, and they were all satisfied with the use of Nursing-Mini-CEX evaluation tool. A total of 63 valid feedbacks were collected during the interview, and the main issues are shown in Figure 3.

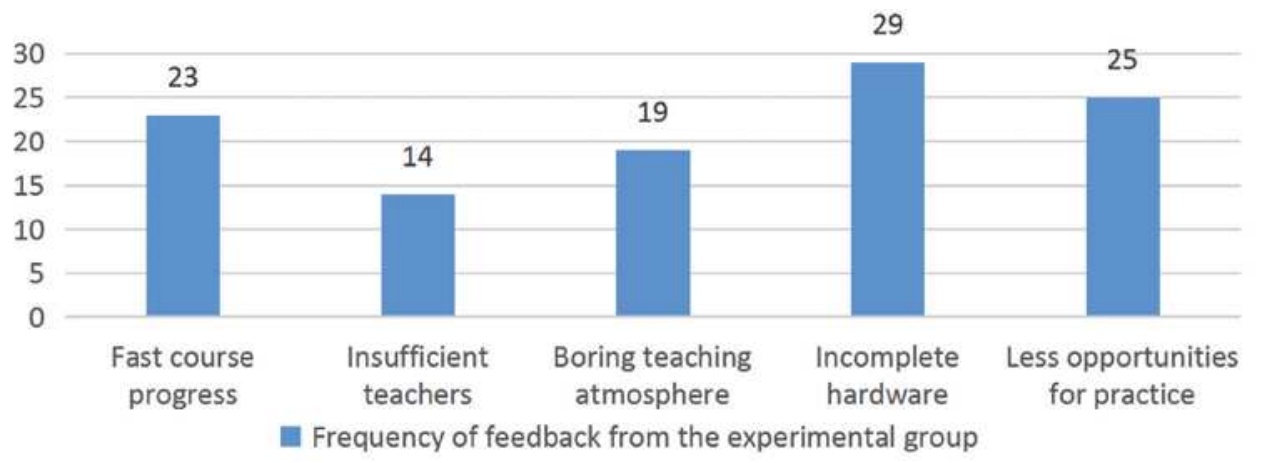

Figure 3. Feedback from the experimental group

\section{Conclusion}

This study found that compared with traditional training evaluation, Nursing-Mini-CEX measurement tool can more accurately reflect the improvement of students' various abilities in basic nursing training. Combining the assessment tool with the basic nursing training of blended teaching can not only promote better learning and targeted improvement of professional abilities, but also promote teachers to better integrate teaching resources. But at the same time, there are still many shortcomings in this research. In the follow-up teaching reform, it should optimize the connection between courses and practical training, and optimize teaching methods and teaching timeliness.

\section{References}

1. Guo Z, Sha L, Yi J, et al. Application of reference system training method in nursing mini-clinical exercise evaluation scorer training. Chinese Nursing Management 2016; 16(09): 1225-1229.

2. Yi J, Sha L, Dong J, et al. Application of Delphi method in compiling nursing mini-clinical drill assessment scale. Nursing Research 2016; 29(14): 1680-1683.

3. Li Y, Wang Q, Zhang Q, et al. Application of miniCEX to standardized training physicians during the rotation of the endocrinology department. Jiangsu Health Service Management 2017; 28(2): 69-70. 